\title{
Interview with Gary Tooze
}

\author{
By Jan Uhde
}

Spring 2010 Issue of KINEMA

\section{INTERVIEW WITH GARY TOOZE OF DVDbeaver.com}

Few people today would fail to see the momentous impact of the DVD medium on the motion pictures - the DVD has changed the way movies are marketed, viewed and above all, revolutionised access to films, both mainstream and art cinema, contemporary and historical, wide-release and niche genres. Still, most reviews and observations on DVD films focus on the disc's actual content - very few of them examine the content's material integrity and technical quality of the transfer, even though the differences, quality and price among individual DVD issues of the same movie can be astounding. Fortunately, there are people who understand the importance of a professional analysis of the material aspects of films on DVD. One is Gary Tooze of Mississauga, Ontario, Canada, who provides exactly such a service on his well-known and widely-appreciated website DVDbeaver.com. Here, Tooze shares his thoughts and insights with Kinema about his project.

KINEMA: When did you launch your site DVDbeaver.com and what inspired you to set it up?

Gary Tooze: I think it was about ten years ago. Initially we were just listing collections, directories, purchases etc. of a small group of like-minded Forum users. We were essentially sharing information with one another. Reviews came later.

Is the DVDbeaver unique or are there similar sites in Canada or anywhere else? If yes, do you see them as competition?

We have a few imitators. There are hundreds of review websites, but none that do the detailed "matching grab" comparisons at anywhere near the volume that we do. I try not to concern myself with what others are doing - we just try to do our best with DVDbeaver.

How many DVDs have you reviewed?

The site has reviewed, or compared, over 6500 DVD or Blu-rays. Personally, I have probably covered about half that.

What are your criteria for selecting a film for your DVD review?

I prefer films that I have enjoyed or that are perceived to have merit and longevity. If there is some viable information to divulge - like exceptional or lackluster quality - we will try to cover that and disseminate these characteristics to our community.

What equipment do you use to analyze DVDs?

The PC does a great job - we use a variety of specific software. I also use my personal home theatre system, especially for the audio. The computer can allow us to zoom in to see grain structure, digital noise, artefacts etc.

How much time does it take to analyze a disc and to compare two discs?

I usually watch the film first on my system and make notes with time references, then use the computer to examine and compare details. A 90-minute movie, with two separate transfers, can take upwards of four hours. We use a consistent methodology so that we can rely on our past findings to help make judgments if we have already reviewed one or more of the editions.

What, in your view, is the most important technical criterion of a quality DVD?

Adherence to the original theatrical appearance and audio - in regards to aspect ratio, cropping, dubbing and lack of digital manipulation. For older films, some companies would inappropriately brighten the image to hide damage marks - this can remove background information as well. 
The illustration below, for example, is from MGM's initial Ingmar Bergman Collection DVD box-set. They used the wrong aspect ratio for the film Shame and cropped the image - look at the chopped head! Our efforts forced a recall of the set and they later re-issued with the correct aspect ratio.

How would you explain to the layman the meaning and importance of the bitrate?

In general terms, the higher the better. More bits of information transferred to your system can translate to a greater level of detail, brighter colours, better sound quality etc. Straight line graphs can represent analog sources, while peaks and valleys are generally a better sign regarding quality.

How do you manage to review so may films - do you have assistants?

I do it full time and we have about half a dozen passionate part-timers.

How do you manage economically? Do you have sponsors?

We obtain a small commission when individuals use our links to purchase from about ten affiliated sources, including all international Amazon online stores and some other Internet retailers. People also donate through PayPal.

Could you name some labels in North America, Europe and elsewhere which have produced the best transfers and top restorations?

Criterion in North America has no real competition in certain areas such as supplementary material. Masters of Cinema, British Film Institute and Second Run in the UK, Studio Canal in France, Kinowelt in Germany and, of course, the major studios are usually consistent, occasionally sneaking out some inferior quality that we are a kind of watchdog for.

Are enough people "out there" aware of what you are doing? Do they appreciate your work? What kind of feedback are you receiving?

We get close to one million hits a month, some very kind and generous supporters. We are always growing. We've had thousands of supportive emails. I really appreciate all the feedback informing us of further details to add to our existing reviews.

Among the classic films which ought to be transferred to DVD is Robert Flaherty's Moana which apparently exists in a sound-added version, a task undertaken in the 1970s by Flaherty's daughter Monica. Do you have an idea why the film was never transferred? (The same question goes for von Stroheim's Greed.)

I am not aware of Moana, my knowledge is often limited to films available on DVD. I think there were a couple of shortened public domain DVDs of Greed - the trouble with that film is having decent elements of the full version - they either don't exist or require extensive restoration. Hopefully it will surface soon - we keep hearing rumours.

Could you name two or three DVDs or DVD restorations which you consider perfect?

There are so many - Criterion's L'eclisse by Antonioni, Olmi's I fidanzati and Fellini's Nights of Cabiria for films on Standard Definition (SD) DVD, Blu-rays of Kubrick's 2001: A Space Odyssey and Bergman's The Seventh Seal, Nicolas Ray's Bigger Than Life, Malick's Days of Heaven; Masters of Cinema's City Girl (Murnau)... more are coming out weekly. The new Blu-rays of the Toy Story films look stunning. I would consider all these "essential."

What films, genres, filmmakers do you like personally?

Antonioni, Bresson, the Dardenne brothers, Tarkovsky, Fellini, Hitchcock, Powell and Pressburger - regarding genres, I enjoy Film Noir the most, westerns, older classic and arthouse. As a diversion I'll watch fifties sci-fi and "creature-feature" films.

Anything else?

Blu-Ray seems to be the future of home cinema. Seeing films in the glory of 1080 Progressive-scan resolution can really change your viewing perceptions and how you can emotionally relate to the film experience. I 
found this is true for so many older films I have watched that I never had the opportunity to see theatrically. Once you look at the improved visuals it's hard to go back. The high-definition shows better detail, brighter, more accurate colours and visible film grain. If you are still considering upgrading, my advice would be "don't hesitate"!

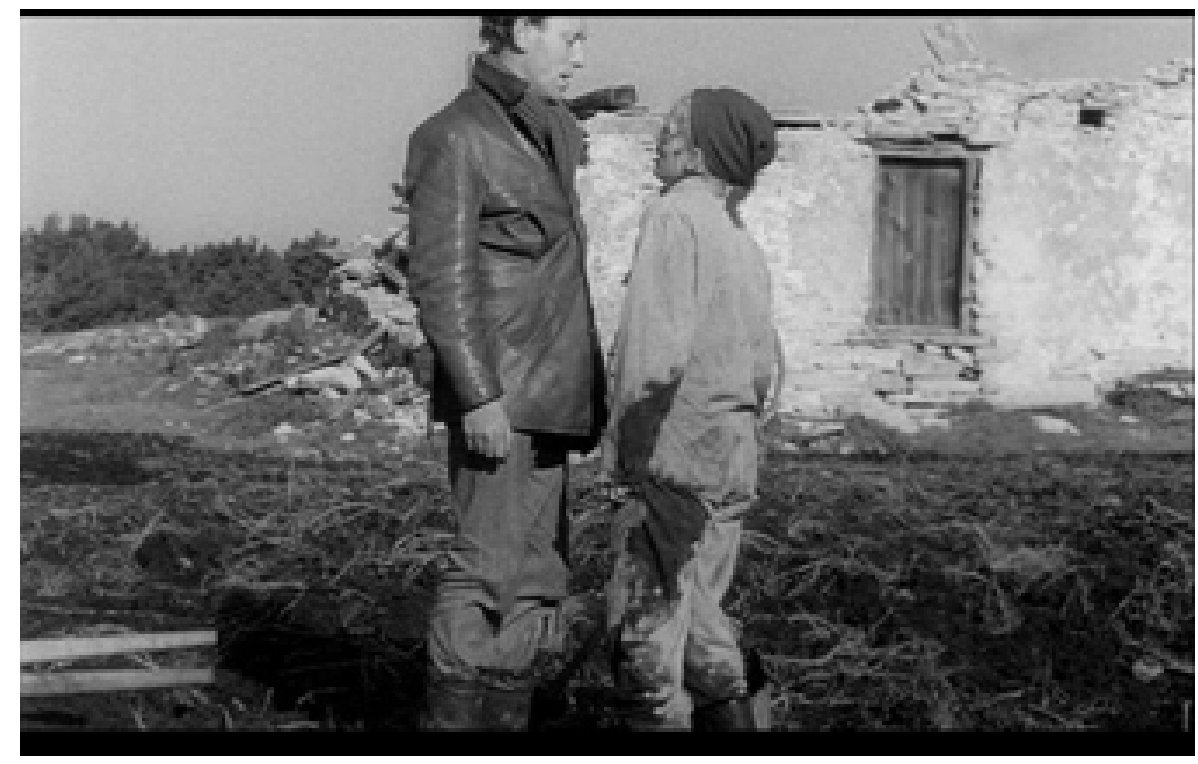

Figure 1: Cropped image from Ingmar Bergman's film Shame.

\section{Author Information}

Jan UHDE is Professor Emer. (Film Studies) at the University of Waterloo, Ontario, Canada. Born in Brno, Czech Republic. Graduated (MA) from the Faculty of Arts, Masaryk University, Brno; PhD received at the University of Waterloo, Ontario, Canada. He taught at the University of Waterloo (1970-2012) where he founded a General and Honours BA program in Film Studies at the Department of Fine Arts.

Publications: Latent Images: Film in Singapore Second edition, with Yvonne Ng Uhde (Ridge Books, National University Press of Singapore, 2010); Latent Images: Film in Singapore, with Yvonne Ng Uhde (Oxford University Press, 2000); Latent Images: Film in Singapore CD-ROM (2003, co-author); Vision and Persistence: Twenty Years of the Ontario Film Institute (University of Waterloo Press, 1990) and Ontario Film Institute Programming Activities Index 1969-1989 (Toronto: Ontario Science Centre, 1990). He co-edited the Place in Space: Human Culture in Landscape (Proceedings from the Second International Conference of the Working Group "Culture and Landscape" of the International Association of Landscape Ecology, Pudoc Scientific Publishers, Wageningen, Holland, 1993). Jan Uhde has published articles and reviews in several countries (including Canada, USA, Germany, Italy), participated in international juries at film festivals and presented papers at international conferences in North America and Europe. In 1998/99, he was a visiting researcher at the School for Film and Media Studies, Ngee Ann Polytechnic, Singapore.

His professional and research interests focus on Singapore cinema; the identification and distancing mechanisms of the film viewer; the non-authored modifications and manipulation of films; and specific aspects of film history, including the Central European cinema.

He founded KINEMA in 1993. 